Apidologie, 1981, 12 (4), 363-376.

\title{
EFFETS COMPARÉS DE DEUX PYRÉTHRINOIDES DE SYNTHÈSE ET DE TROIS INSECTICIDES ORG ANOPHOSPHORÉS SUR LES MÉGACHILES (Megachile rotundata F. = pacifica Pz.)
}

\author{
J.-N. TASEI et P. DINET \\ Laboratoire de Zoologie - I.N.R.A. - 86600 Lusignan
}

\begin{abstract}
RÉSUMÉ
Les auteurs ont testé sur des mégachiles mâles (Megachile rotundata $\mathrm{F}$.) la toxicité de 5 produits commerciaux à base de phosalone, bromophos, oxydémeton-methyl, decaméthrine et fenvalérate. Les tests avec papier à chromatographie ou plante traitée ainsi que la pulvérisation directe sur les insectes montrent que le bromophos est très toxique ( 85 à $100 \%$ de mortalité). Selon le type de test la phosalone cause des pertes variant de 52 à $80 \%$, la décaméthrine de 5 à $95 \%$, l'oxydéméton-méthyl de 2 à $40 \%$ et le fenvalérate de 3 à $27 \%$. Chez les pyréthrinoïdes : fenvalérate et décaméthrine on observe des comas réversibles suivis de détoxication, concernant jusqu'à $60 \%$ de la population testée.

En serre les femelles de mégachiles manifestent de la répulsion durant $1 \mathrm{~h} 15 \mathrm{mn}$ à $3 \mathrm{~h} 30 \mathrm{mn}$ pour les fleurs de Melilotus alba traitées avec respectivement du fenvalérate et de la décaméthrine.
\end{abstract}

\section{INTRODUCTION}

Si l'on se réfère à l'article de BournoviLle (1979) on comprend pourquoi les luzernes porte-graines ne sont pas suffisamment protégées par les interventions phytosanitaires actuelles à base de phosalone contre les attaques de la cécidomyie des fleurs (Contarinia medicaginis Kief). Les infestations de ce ravageur à vie très brève ( 48 heures) sont en effet caractérisées par le renouvellement incessant des individus qui menacent les jeunes boutons floraux pendant les trois semaines du vol. Seuls des insecticides très rémanents et agissant rapidement peuvent réduire notablement les dégâts. Quelques matières actives nouvelles possèdent les qualités requises pour lutter efficacement contre les. cécidomyies (DELAUDE, 1981), ce sont les pyréthrinoïdes de synthèse dont les molécules, proches de celles des pyréthrines naturelles sont photostables, à haut pouvoir insecticide et très peu solubles dans l'eau (Elloto et al., 1978). 
Cependant l'utilisation de tels produits sur les luzernières ne doit être préconisée que si l'on démontre leur innocuité non seulement pour les abeilles domestiques, mais aussi pour les abeilles solitaires qui pollinisent la luzerne. Dans le présent travail on considère que les mégachiles $(M$. rotundata $F$.) peuvent faire office de représentants de la faune des abeilles solitaires. C'est une espèce dont on peut obtenir un grand nombre d'individus dans des conditions standard et par ailleurs la profession des producteurs de semences fourragères commence à pratiquer en France, dans quelques champs pilotes, les élevages de mégachiles, à partir d'une population importée du Manitoba (Canada) et il est indispensable de protéger ces abeilles contre tout traitement pouvant mettre leur vie en danger.

Dans les pages qui suivent le lecteur constatera que les techniques des tests de contact soit tarsal avec papier traité ou sur plante traitée, soit par pulvérisation, sont inspirées de celles qui étaient proposées dans des publications antérieures (TASEI, 1977; TASEI et al., 1977). Un type nouveau de test a été mis en œuvre pour mesurer l'effet répulsif des pyréthrinoïdes.

Au total cinq produits commerciaux ont fait l'objet de tests de toxicité par contact; ils étaient à base d'organophosphorés : phosalone, bromophos et oxydémétonméthyl et de pyréthrinoïdes de synthèse : décaméthrine et fenvalérate.

\section{MATÉRIEL ET MÉTHODES}

\section{$I^{\circ}$ Obtention des insectes}

Le travail est accompli presque uniquement sur les mâlesłale l'espèce pour des raisons méthodologiques : les femelles résistent moins bien à la claustration que les mâles, mais aussi pour des raisons économiques : les mâles sont 2 à 3 fois plus nombreux que les femelles.

Quelques milliers de cocons obtenus en champ sont placés en étuve à $29^{\circ} \mathrm{C}$ et à environ $60 \%$ d'hy grométrie relative. Les individus mâles, émergeant avant les femelles, sont retirés de l'incubateur lors de chacune des 3 à 4 inspections journalières et sont placés à $17^{\circ} \mathrm{C}$ environ dans une salle obscure. Au bout de 24 à 48 heures le nombre d'individus requis pour un test est atteint et à ce moment le reste des cocons est stocké à $17^{\circ} \mathrm{C}$ jusqu'à ce qu'une nouvelle expérience exige la reprise de l'incubation.

\section{$2^{o}$ Test de contact avec les résidus sur papier}

Ces tests se déroulent dans une enceinte climatisée à $27^{\circ} \mathrm{C} \pm 1,55 \%$ d'humidité relative et éclairée par des tubes fournissant 1100 lux. Les insectes mâles sont enfermés sans nourriture durant 26 heures dans des cagettes en polystyrène cristal de $15 \times 10 \times 2 \mathrm{~cm}$ à couvercle grillagé. Avant l'introduction des mégachiles on dépose sur le fond une feuille de papier à chromatographie Whatman pesant $185 \mathrm{~g} \mathrm{au} \mathrm{m}$. Cette feuille est imprégnée d'une quantité connue d'insecticide (tableau 1). Des essais préliminaires ont permis de préciser que le volume nécessaire à l'imbibition totale des feuilles de $150 \mathrm{~cm}^{2}$ sans ressuyage est de $4 \mathrm{ml}$, déposés de manière homogène à la pipette. Ce volume équivaut à $2700 \mathrm{I}$ de solution/ha. Les dilutions des insecticides sont calculées sur cette base de sorte que la quantité de produit par unité de surface sur le papier soit égale à celle des doses à l'hectare choisies pour les autres types de test. Les papiers traités sont introduits en cage dès qu'ils sont secs.

Les mégachiles restent en permanence au contact du papier et ne se déplacent qu'au fond de la boite. Chaque traitement est répété 4 fois et chaque boite contient 16 individus. On effectue des comptages de mortalité toutes les heures pendant les six premières heures, puis les intervalles de comptage sont plus longs. 
TABL. 1. - Dilutions des insecticides utilisées pour les tests de toxicité par contact sur papier à chromatographie.

TABL. 1. - Dosages of insecticides utilized in the residual contact test on chromatography paper.

\begin{tabular}{|c|c|c|c|c|c|}
\hline Matière active & $\begin{array}{c}\text { Produit } \\
\text { commercial } \\
\text { Commercial } \\
\text { compound }\end{array}$ & $\begin{array}{l}\text { Poids de matière } \\
\text { active par } \\
\text { hectare } \\
\text { Weight of active } \\
\text { ingredients/ha }\end{array}$ & $\begin{array}{l}\text { Matière active } \\
\text { par litre } \\
\text { de produit } \\
\text { commercial } \\
\text { Active ingredients } \\
\text { /l of commercial } \\
\text { compound }\end{array}$ & $\begin{array}{c}\begin{array}{c}\text { Dilution utilisée } \\
\text { (Matière active) }\end{array} \\
\begin{array}{c}\text { Dosage (active) } \\
\text { ingredients }\end{array}\end{array}$ & $\begin{array}{l}\text { Dilution utilisée } \\
\text { produit } \\
\text { commercial } \\
\text { Dosage } \\
\text { (commercial } \\
\text { compound }\end{array}$ \\
\hline Phosalone & Zolone liquide & $1000 \mathrm{~g} / \mathrm{ha}$ & $350 \mathrm{~g} / 1$ & $0,370 \mathrm{~g} / \mathrm{l}$ & $1,058 \mathrm{ml} / \mathrm{l}$ \\
\hline Bromophos & Sovi Nexion 25 & $500 \mathrm{~g} / \mathrm{ha}$ & $250 \mathrm{~g} / 1$ & $0,185 \mathrm{~g} / 1$ & $0,740 \mathrm{ml} / 1$ \\
\hline $\begin{array}{l}\text { Oxydéméton- } \\
\text { méthyl }\end{array}$ & Métasystemox $\mathrm{R}$. & $250 \mathrm{~g} / \mathrm{ha}$ & $250 \mathrm{~g} / 1$ & $0,092 \mathrm{~g} / 1$ & $0,370 \mathrm{ml} / 1$ \\
\hline Décaméthrine & Décis & $7,5 \mathrm{~g} / \mathrm{ha}$ & $25 \mathrm{~g} / 1$ & $0,0027 \mathrm{~g} / 1$ & $0,111 \mathrm{ml} / 1$ \\
\hline Fenvalérate & Sumicidin 10 & $50 \mathrm{~g} / \mathrm{ha}$ & $100 \mathrm{~g} / 1$ & $0,0185 \mathrm{~g} / 1$ & $0,185 \mathrm{ml} / \mathrm{l}$ \\
\hline
\end{tabular}

Une variante de ce test de base, consiste à transférer les mâles après 4 heures de contact, dans des bốtes propres et à poursuivre les comptages.

\section{$3^{\circ}$ Test de contact par pulvérisation}

Ce test, qui a lieu en après-midi, simule un traitement insecticide en période de vol. Les mâles sont introduits dans les mêmes boites que précédemment. Au fond de celles-ci on dispose deux feuilles de papier à chromatographie de façon à absorber rapidement l'excès de liquide insecticide qui pourrait entraíner un surdosage. Les traitements sont effectués sur 2 boites contenant chacune 30 mâles, déposées par terre sur le passage de la rampe du pulvérisateur "Pulval n (1). Ce pulvérisateur porté à dos d'homme, à jet projeté par air comprimé (pression 3 bars) répand 1 litre de liquide sur $20 \mathrm{~m}^{2}$ soit l'équivalent de 500 litres à l'hectare. La rampe de l'appareil est réglée à une hauteur de $50 \mathrm{~cm}$. Le traitement a lieu à une température de $18^{\circ} \mathrm{C}$. Dès que la pulvérisation est achevée les insectes sont retirés des boites et introduits dans des cages grillagées de "récupération" de $1,60 \times 0,60 \times 0,50 \mathrm{~m}$ contenant un pied de mélicot blanc (Melilotus alba) fleuri. Ces cages sont rangées côte à côte dans une serre vitrée dans laquelle les températures diurnes ne dépassent pas $34^{\circ} \mathrm{C}$ et les températures nocturnes ne sont pas inférieures à $10^{\circ} \mathrm{C}$. Le comptage des morts se fait aux mêmes intervalles de temps que dans le test précédent. Les concentrations des solutions en matière active et en produit commercial sont indiquées dans le tableau 2.

\section{$4^{\circ}$ Test de contact avec des plantes traitées}

On traite au pulvérisateur Pulval des pieds de melilot cultivés en caissettes de terreau, de la même manière que dans le test décrit plus haut. Dès que le feuillage est sec on introduit les plantes et les insectes dans des cages grillagées de $1,5 \mathrm{~m}$ de haut et de $1,20 \times 0,75$ de base. Un film plastique recouvre le côté exposé au vent dominant ainsi que le toit, afin d'éviter le lessivage des résidus par la pluie. Chaque traitement est répété 2 fois. Chaque cage contient 40 mâles. On compte régulièrement les morts pendant 10 jours. Durant les 5 premiers jours les températures maximales ont oscillé entre 17 et $23^{\circ} \mathrm{C}$.

\section{$5^{\circ}$ Test de répulsion par les 2 pyréthrinoïdes}

On a procédé à ce test dans 2 compartiments de serre contigus pouvant chacun contenir une trentaine de pieds de mélilot blanc en fleurs. On libère dans chaque compartiment une quarantaine de femelles accompagnées de mâles afin qu'il y ait accouplement. Lorsque toutes les femelles sont en cours de nidifica-

(1) Spécialité de la maison Cristal. 
TABL. 2. - Dilutions des insecticides utilisées pour les tests par pulvérisation directe ou sur plantes traitées.

TABL. 2. - Dosage of insecticides utilized in the spraying test or the residual contact test on treated plants.

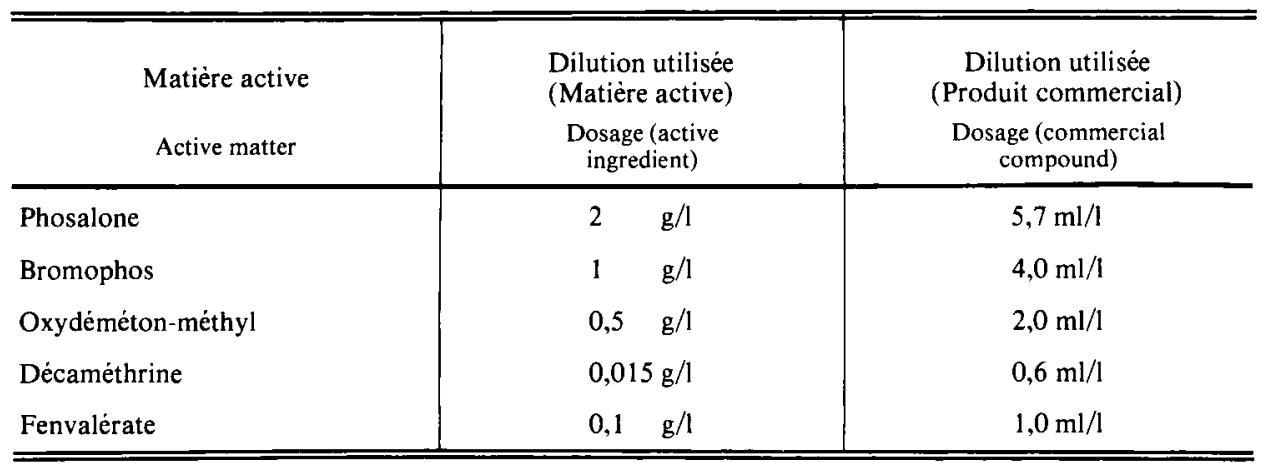

tion on compte 10 fois alternativement dans les 2 compartiments le nombre de femelles butinant instantanément soit en moins d' $1 \mathrm{mn}$ sur les 30 plantes de mélilot. Puis la moitié des plantes de chaque compartiment sont sorties et traitées avec de la décaméthrine ou du fenvalérate, comme dans les tests précédents. Ces plantes sont replacees respectivement dans les deux compartiments. A partir de ce moment on effectue à intervalles de temps réguliers une dizaine de comptages " instantanés " sur les femelles butinant les plantes traitées et les plantes témoins. Cette mesure de la répulsivité commence $15 \mathrm{mn}$ après le traitement.

\section{RÉSULTATS}

$1^{o}$ Toxicité par le test de contact avec du papier traité (Fig. 1 et 2).

Les résultats de comptages ont permis de dresser les courbes de la figure 1. Celles qui représentent l'évolution de la mortalité en présence des pyréthrinoïdes appellent un commentaire sur l'effet de " knock down " de ces substances. La réaction des mégachiles à la décaméthrine et au fenvalérate est très rapide puisqu'une heure après l'introduction des insectes, 30 à $40 \%$ d'entre eux sont sur le dos, presqu'immobiles ou tremblant rythmiquement. Ils sont tous comptés comme morts alors qu'un certain nombre d'entre eux ne sont que dans un état comateux réversible. En effet la poursuite des observations au cours de la troisième heure et des suivantes montre qu'une partie de ces individus se sont détoxiqués et ont repris une activité normale. Les mortalités indiquées pour ce test ainsi que pour les tests suivants incluent donc les individus comateux.

Le test effectué sur papier avec contact permanent indique un effet de choc (knock down) presqu'aussi puissant chez le fenvalérate et la décaméthrine mais une détoxication beaucoup plus lente chez cette dernière, soit en 20 heures au lieu de 10 heures chez le fenvalérate. Cette détoxication porte sur $44 \%$ des mégachiles traitées à la décaméthrine et $59 \%$ des mégachiles traités au fenvalérate.

Le test sur papier avec un contact des insectes limité à 4 heures (Fig. 2) montre que l'effet de choc et la détoxication du fenvalérate et de la décaméthrine sont identiques. 


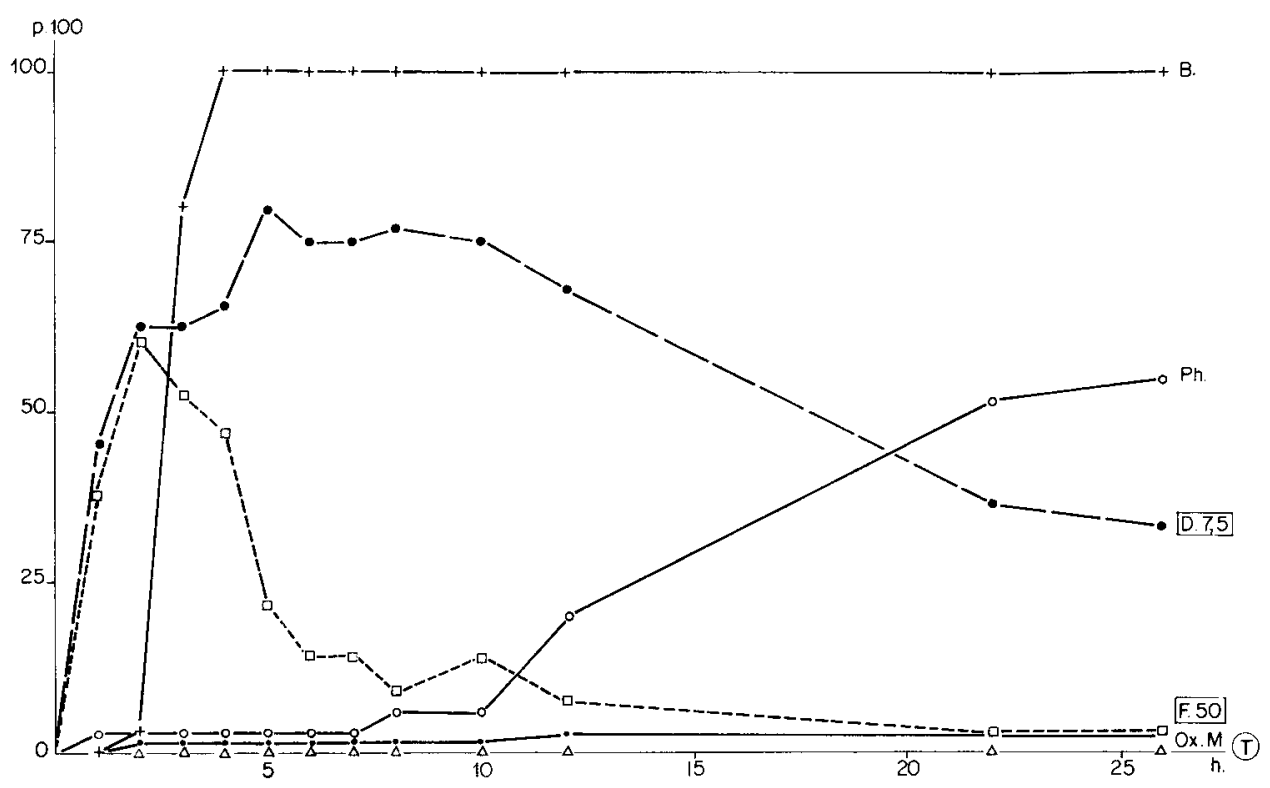

FIG. 1. - Toxicité de 5 insecticides pour des mâles de mégachiles. Test par contact permanent avec papier traité.

Verticalement : pourcentage de morts et comateux.

Horizontalement : durée du test en heures.

$\mathrm{B}=$ Bromophos.

$\mathrm{Ph}=$ Phosalone.

$\mathrm{D}(7,5)=$ Décaméthrine $(7,5 \mathrm{~g}$ de matière active à l'hectare).

$\mathrm{F}(50)=$ Fenvalérate $(50 \mathrm{~g}$ de matière active à l'hectare).

Ox.M $=$ Oxydéméton-méthyl.

$\mathrm{T}=$ Témoin.

N.B. : la réversibilité de certains comas est signalée par les parties descendantes des courbes D. 7,5 et F. 50 .

FIG. 1. - Toxicity of 5 insecticides to males of megachiles.

Test by residue exposure on treated paper.

$\mathrm{y}$ axis $=\%$ dead and comatose bees.

$\mathrm{x}$ axis $=$ Lenght of test in hours.

$\mathrm{t}=$ control.

Les deux tests prouvent que si la rapidité d'action est très grande chez les pyréthrinoïdes, elle est un peu plus faible chez le bromophos et très faible chez la phosalone. On peut classer les produits par ordre de toxicité décroissante au bout de 24 heures : bromophos, phosalone, décaméthrine, fenvalérate, oxydéméton-méthyl.

\section{$2^{o}$ Toxicité par pulvérisation directe (Fig. 3)}

Le classement par la rapidité d'action est le même que lors des tests par contact avec du papier. 


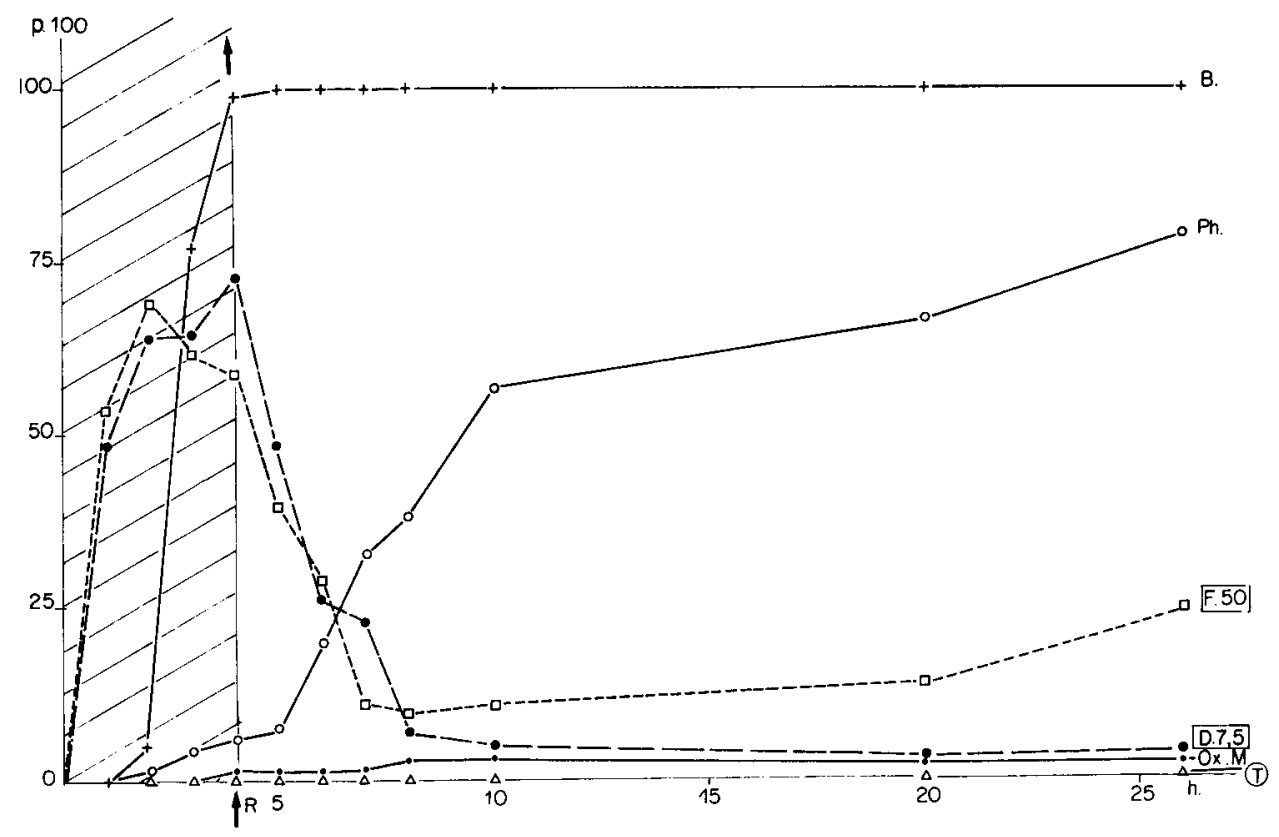

FIG. 2. - Toxicité de 5 insecticides pour des mâles de mégachiles.

Test par contact de 4 heures avec papier traité.

$\mathbf{R}=$ Retrait du papier traité.

Même légende que Fig. 1.

FIG. 2. - Toxicity of 5 insecticides to males of megachiles.

Test of 4 hours exposure on treated paper.

$\mathrm{R}=$ Paper is with-drawn

Same caption as Fig. 1 .

Si l'on considère la mortalité des mâles au bout de 24 heures, les substances les plus dangereuses sont le bromophos et la décaméthrine tandis que la phosalone, l'oxydéméton-méthyl et le fenvalérate sont moyennement toxiques.

La détoxication s'opère en 14 heures chez le fenvalérate et concerne $62 \%$ du total des individus alors qu'elle est très peu sensible chez la décaméthrine puisqu'elle ne porte que sur $5 \%$ des individus.

\section{$3^{\circ}$ Toxicité par contact avec des plantes traitées (Fig. 4)}

Si l'on considère le cinquième jour de butinage sur les mélilots, c'est-à-dire le moment où les taux de mortalité n'évoluent plus, on voit que 3 substances sont très toxiques: le bromophos, la phosalone et la décaméthrine. L'oxydéméton-méthyl est moyennement toxique et le fenvalérate faiblement toxique.

La rapidité d'action du bromophos et du fenvalérate est évidente, la toxicité de la phosalone est surtout de l'oxydéméton-méthyl et de la décaméthrine ne se manifeste que tardivement au cours du deuxième jour. 


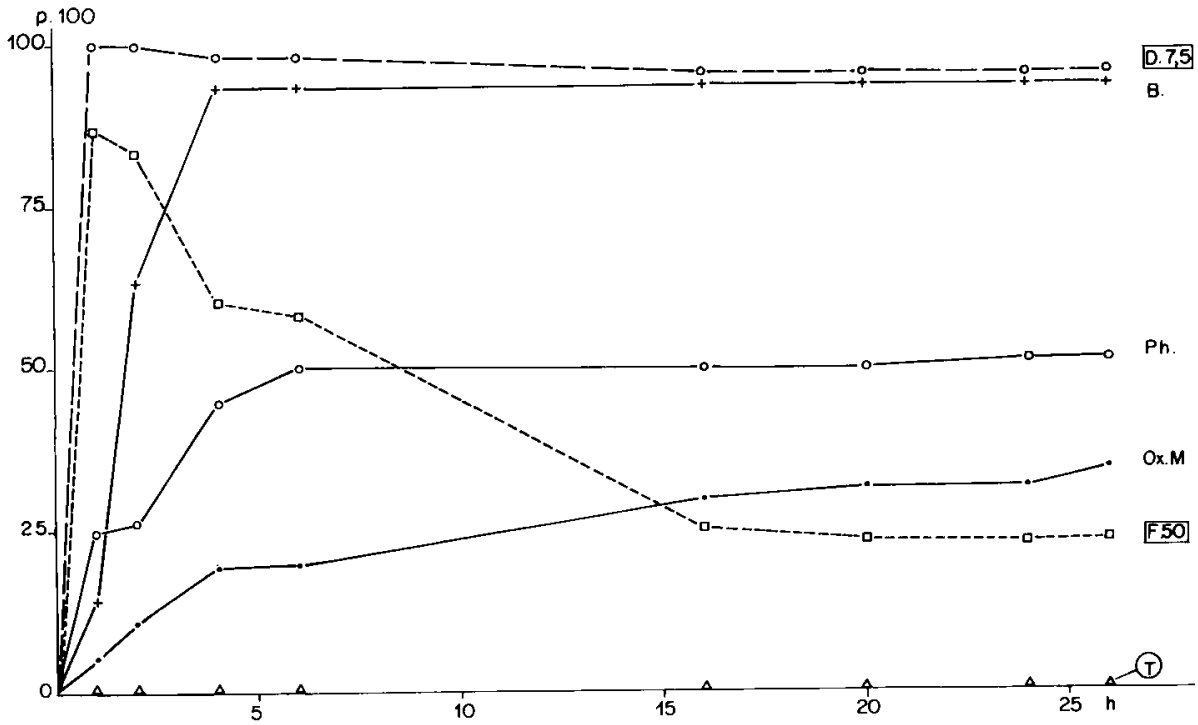

FIG. 3. - Toxicité de 5 insecticides pour des mâles de mégachiles. Test par pulvérisation directe.

Même légende que Fig. 1.

FIG. 3. - Toxicity of 5 insecticides to males of megachiles.

Test by direct spray on bees.

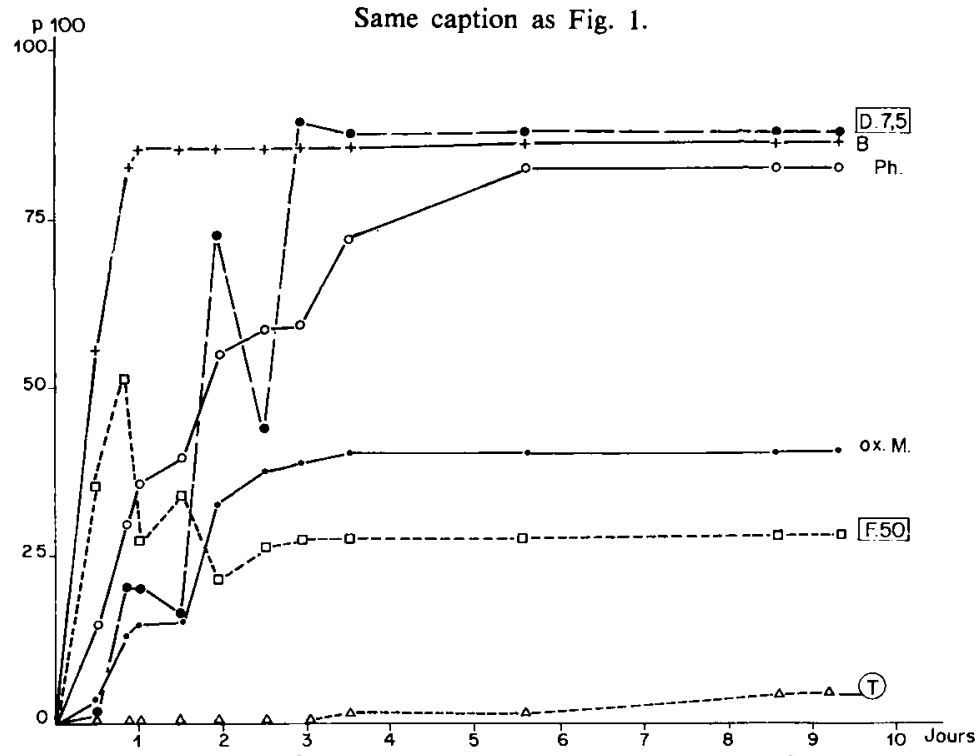

Fig. 4. - Toxicité de 5 insecticides pour des mâles de mégachiles. Test avec les plantes de melilot (Melilotus alba) traitées.

Même légende que Fig. 1

FIG. 4. - Toxicity of 5 insecticides to males of megachiles. Test with treated sweet clover plants (Melilotus alba). $\mathrm{x}$ axis $=$ lenght of test in days

Same caption as Fig. 1.

Horizontalement : Durée du test en jours. 


\section{$4^{o}$ Test de répulsivité}

Ce test n'a été pratiqué que sur les 2 pyréthrinoïdes. Les 10 comptages alternés dans chaque compartiment de serre sur les plantes non traitées (tableau 3) montrent que les deux substances sont répulsives. Les femelles s'approchent à $1 \mathrm{~cm}$ des fleurs mais généralement ne se posent pas dessus. Lorsqu'elles entrent en contact avec elles leur passage est très furtif. Cette répulsion dure $1 \mathrm{~h} 15 \mathrm{mn}$ chez le fenvalérate et $3 \mathrm{~h} 30 \mathrm{mn}$ chez la décaméthrine dans les conditions de serre. Le tableau 3 indique en outre que l'introduction des plantes traitées ne provoque pas un accroissement du butinage des plantes témoins.

\section{DISCUSSION - CONCLUSION}

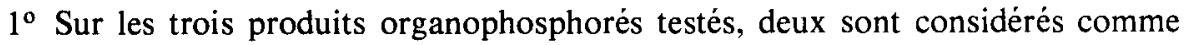
non toxiques pour l'Abeille domestique (phosalone et bromophos). Les expériences ont montré qu'aux doses utilisées et dans les conditions de test, la phosalone est moyennement ou fortement dangereuse pour les mâles de mégachiles. Quant au bromophos il est toujours très dangereux (tableau 4). Par contre l'oxydéméton-méthyl considéré comme toxique pour l'Abeille domestique se révèle une fois de plus faiblement ou moyennement toxique selon le test (TASEI et al., 1977).

Le fenvalérate à $50 \mathrm{~g}$ de matière active à l'hectare n'occasionne, selon le type de test, que des mortalités moyennes ou très faibles. Les effets de la décaméthrine à $7,5 \mathrm{~g}$ de matière active à l'hectare sont beaucoup plus variables que ceux du fenvalérate puisque les mortalités qui lui sont imputables sont soit très faibles, soit très élevées. Il est donc difficile d'opérer un classement définitif des 5 produits mais on peut tout de même dire à partir de nos résultats que les traitements en cours de floraison au fenvalérate et à l'oxydéméton-méthyl sont ceux qui présentent le moins de risques pour les mégachiles.

$2^{\circ}$ Le type d'action est différent selon la substance employée : le bromophos agit très vite, les pyréthrinoïdes également. Dans les conditions de nos tests l'intoxication prend le pas sur la détoxication chez la décaméthrine, ce qui se traduit par un taux de comas réversibles plus réduit que chez le fenvalérate. La phosalone manifeste tardivement sa toxicité et l'on peut supposer que seuls agissent les métabolites de cette matière active après sa pénétration. L'oxydéméton-méthyl n'a d'action bien perceptible que si le substrat traité est une plante en fleurs. La toxicité qui se déclare alors quelques jours après le traitement est certainement due aux propriétés "systémiques " du produit qui finit par être véhiculé par le nectar à des doses létales ou sub-létales pour les mégachiles.

La répulsion temporaire observée chez un certain nombre de femelles de mégachiles devant les mélilots traités aux pyréthrinoïdes a été signalée chez l'Abeille domes- 
MEGACHILES ET PYRETHRINOÏDES DE SYNTHÈSE

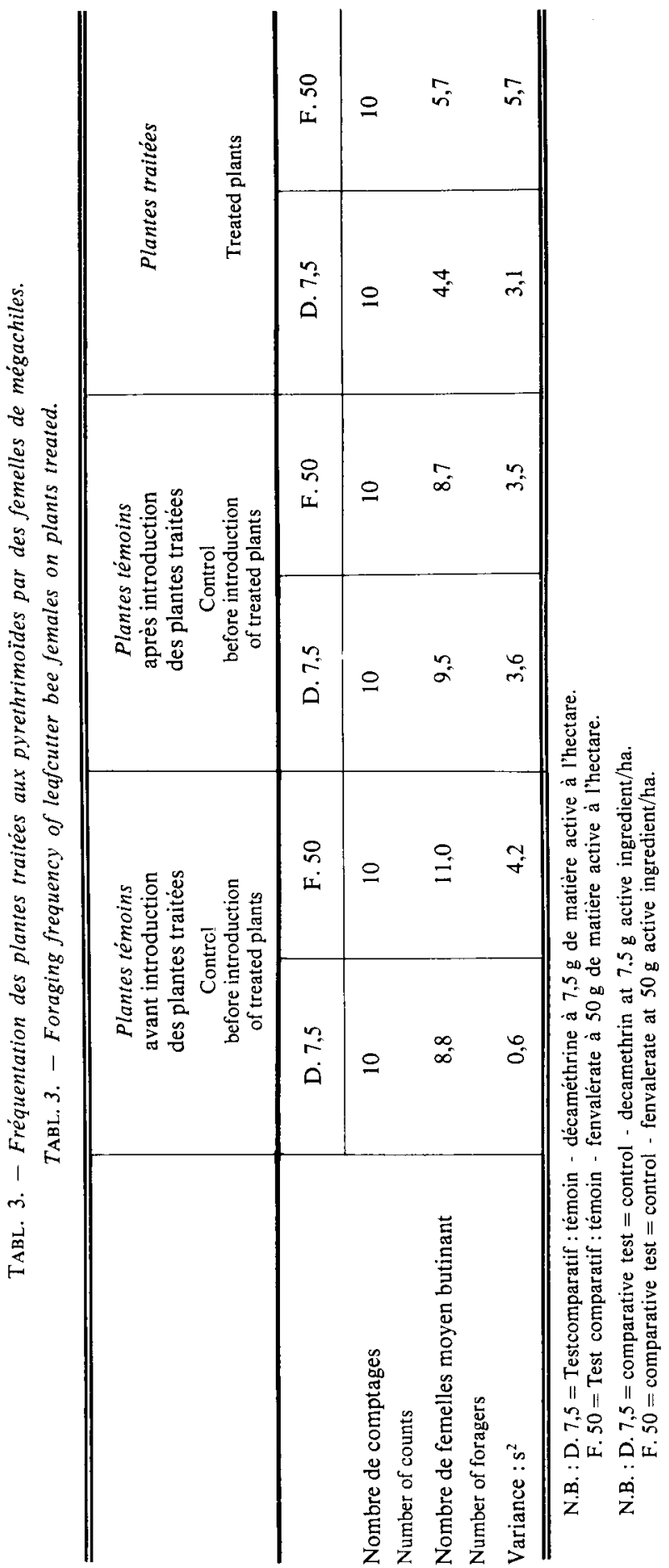




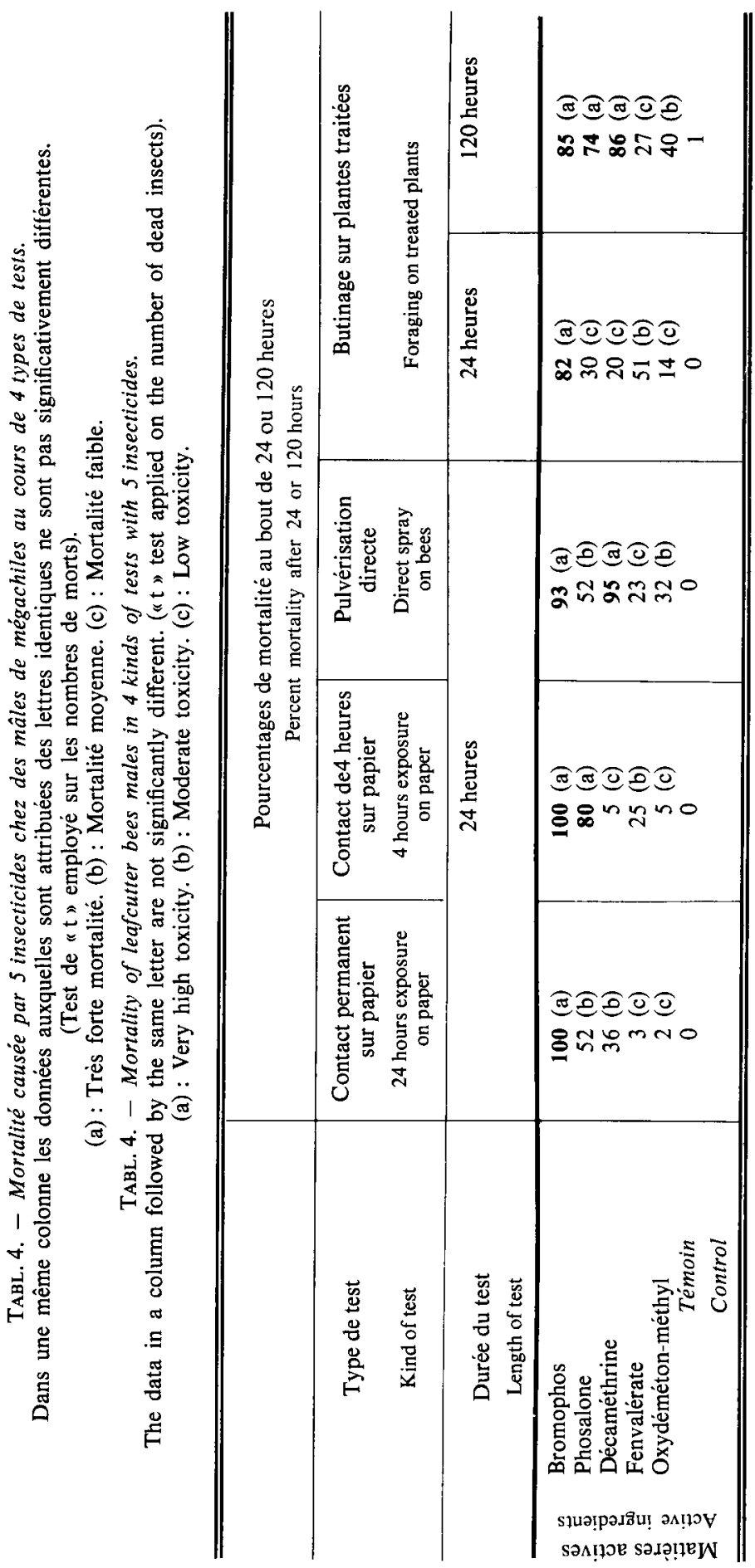


tique par plusieurs auteurs (Gerig, 1979; Cazenave et al., 1980) et dans plusieurs conditions expérimentales (cages, cagettes, tunnel, plein air). Chez cette espèce la répulsion est beaucoup plus forte et de plus longue durée. Ce phénomène est à rapprocher de l'effet anti-appétant des pyréthrinoïdes chez certaines chenilles (LHOste, 1978).

$3^{\circ}$ Les travaux que nous venons d'exposer confirment que les mégachiles de la luzerne ( $M$. rotundata) sont un bon matériel biologique pour exécuter des tests de laboratoire ou des tests en conditions semi-naturelles.

La concordance relativement bonne des résultats des divers tests permet un classement des produits commerciaux selon leur toxicité. Cependant la généralisation des premières conclusions concernant les pyréthrinoïdes, à l'échelle de la pratique agricole demande des informations supplémentaires. Aussi les études ultérieures devront répondre aux questions suivantes :

a) Comment varie la sensibilité des mâles et des femelles de mégachiles en fonction de leur âge? Cette interrogation est suggérée par JOHANSEN (1979) qui affirme qu'après 3 semaines de vol les mégachiles de la luzerne sont plus sensibles aux insecticides que ceux qui émergent.

b) Comment réagissent les femelles aux traitements? En particulier, quelles sont les conséquences de l'effet de choc et sa répétition éventuelle chez la femelle en cours de nidification? Il est important de savoir si les capacités de vol et de repérage du nid, après récupération, ne sont pas plus affectées en champ qu'en milieu clos.

c) L'effet répulsif, même s'il ne dure que peu de temps, peut-il troubler les facultés de nidification des femelles?

d) Quelle est la rémanence des produits testés, particulièrement les pyréthrinoïdes?

\section{REMERCIEMENTS}

Les échantillons de produits commerciaux ont été donnés par les firmes : Procida, Bayer, Sovilo, Rhodiagri et Agrishell. Le financement d'une partie de cette étude est dû à cette dernière firme que nous remercions.

Reçu pour publication en mai 1981.

Eingegangen im Mai 1981.

\section{ZUSAMMENFASSUNG}

VERGLEICH DER WIRKUNG VON ZWEI SYNTHETISCHEN PYRETHRINOIDEN UND DREI ORGANOPHOSPHOR-INSEKTIZIDEN AUF MEGACHILIDEN

(MEGACHILE ROTUNDA $\mathrm{F} .=$ pacifica $\mathrm{Pz}$.)

Der Befall der Saatzucht-Felder der Luzerne durch die blütenzerstörende Cecidomyide Contarinia medicaginis K. ist durch Mittel vom Typ des Phosalons nur schwer zu beherrschen (BourNoviLle, 1979). Die synthetischen Pyrethrinoide, von grosser insektizider und von langandauernder Wirkung, 
gewährleisten einen besseren Schutz der Pflanzen. Ihr Einsatz in grösserem Stil kann jedoch nur dann empfohlen werden, wenn sie weder für Honigbienen noch für solitäre Bienen toxisch sind. Wir glauben, dass die Art Megachile rotundata F. als Repräsentant der letzteren gelten kann, und dies umsomehr, als man jetzt auch in Frankreich beginnt, diese Biene für den Einsatz in der Luzerne-Bestäubung zu halten.

\section{Material und Methoden}

Es wurden fünf Präparate einem Toxiditätstest unterzogen. Sie waren aus der Gruppe von Phosalon, Bromophos, Methyl-Oxydemeton, Decamethrin und Fenvalerat.

Nur Megachile-Männchen wurden in den Experimenten eingesetzt (in kleineren oder grösseren Käfigen).

In der ersten Versuchsanordnung wurden $16 \times 4$ Individuen mit Blättern von ChromatographiePapier in Kontakt gebracht, die mit Insektizid-Lösungen getränkt waren; die Konzentrationen sind in Tab. 1 angegeben. Die Versuchskäfige mit den Massen $15 \times 10 \times 2 \mathrm{~cm}$ wurden für 24 Stunden in einem mit 1.100 lux beleuchteten Käfig bei $27 \pm 1^{\circ} \mathrm{C}$ gehalten.

Bei der zweiten Versuchsanordnung wurde die Insektizid-Lösung auf die Blattschneidebienen zerstäubt. Diese wurden dann in Käfige mit Melilotus-Pflanzen (Melilotus albus) eingesetzt. Die benutzten Konzentrationen sind in Tab. 2 angegeben.

Bei der dritten Versuchsanordnung wurde die Insektizid-Lösung auf die Melilotus-Pflanzen versprüht; nach dem Abtrocknen wurden sie zusammen mit Megachile-Männchen in Käfige von $1,50 \times 1,20 \times 0,75 \mathrm{~m}$ eingesetzt.

Ein Test über die Reppellent-Wirkung wurde mit Megachile-Weibchen durchgeführt. Er erfolgte in einem Glashaus mit Melilotus, von dem die eine Hälfte behandelt wurde - entweder mit Decamethrin oder mit Fenvalerat.

\section{Resultate}

- Die Schockwirkung, der " knock down ", ist bei Fenvalerat fast ebenso stark wie bei Decamethrin. Bei letzterem erholten sich $44 \%$ der Tiere aber wieder, gegen $59 \%$ bei Fenvalerat (Fig. 1). Wurde das behandelte Papier nach 4 Stunden entfernt (Fig. 2), so sind Schockwirkung und Erholung (Detoxikation) bei beiden Pyrthrinoiden identisch. Die Fig. 1 und 2 gestatten eine Klassifizierung der Präparate nach abnehmender Toxidität : Bromophos, Phosalon, Decamethrin, Fenvalerat, Methyl-Oxydemeton.

- Bei direkter Bestäubung (Fig. 3) liegt die Geschwindigkeit der schädigenden Wirkung der Präparate in derselben Grössenordnung wie bei den vorangegangenen Versuchen. Gefährliche Präparate sind das Bromophos und das Decamethrin. Phosalon, Methyl-Oxydemeton und Fenvalerat sind von mittlerer Toxidität. Die Detoxikation ist besonders ausgeprägt bei Fenvalerat (62\%).

- Am fünften Tag der Sammeltätigkeit im Käfig (Fig. 4) sind drei Substanzen sehr toxisch : Bromophos, Phosalon und Decamethrin. Methyl-Oxydemeton und Fenvalerat liegen etwa in der Mitte und sind nur wenig toxisch.

- Die Repellentwirkung der mit den Pyrethrinoiden behandelten Pflanzen zeigt sich unter den Bedingungen des Glashauses bei Fenvalerat über 1 st $15 \mathrm{~min}$, bei Decamethrin über 3 st 30 min (Tab. 3).

- Die Megachile-Männchen sind sehr oder ziemlich empfindlich gegenüber zwei Substanzen, die bisher als nicht toxisch für Honigbienen galten (Bromophos und Phosalon); Methyl-Oxydemeton hingegen ist relativ wenig toxisch.

- Die Pyrethrinoide lassen sich als wenig bis sehr gefährlich klassifizieren wie das Decamethrin, oder als schwach mittelgefährlich wie Fenvalerat - je nach der Anwendung (Tab. 4).

- Methyl-Oxydemeton scheint nicht gefährlich zu sein, sofern es mit dem Nektar übertragen wird.

- Die Schockwirkung der Pyrethrinoide könnte zu Störungen beim Nestbau der Megachile führen, selbst wenn nachher eine Detoxikation erfolgt. Es sollten auf diesem Gebiete noch weitere Untersuchungen durchgeführt werden. 
- Es sind vergleichende Untersuchungen der Empfindlichkeit der Männchen und der Weibchen unter gleichen Testbedingungen geplant.

\author{
SUMMARY \\ COMPARISON OF THE EFFECTS OF 2 SYNTHETIC PYRETHROIDS \\ AND 3 ORGANOPHOSPHOROUS INSECTICIDES \\ ON THE ALFALFA LEAFCUTTER BEE (MEGACHILE ROTUNDATA F. = PACIFICA Pz.)
}

Attacks of lucerne seed fields by flower gall midges (Contarinia medicaginis K.) are not adequately controlled by insecticides similar to phosalone (Bournoville, 1979). Synthetic pyrethroids are very toxic and persistent but they ensure better plant protection. Their large scale utilization might be recommended only if they prove to be harmless to honey bees and solitary bees. We believe Megachile rotundata $\mathrm{F}$. could be the standard solitary bee, as rearing of this species is increasing in France.

\title{
Material and methods
}

Five compounds were tested, they were : phosalone, bromophos, oxydemeton-methyl, decamethrin and fenvalerate. Only males of megachile were submitted to tests in cages or boxes.

In the first test, repeated 4 times, 16 bees were exposed to residues on chromatography papers previously saturated with levels of insecticides as shown in table 1 . The volume of solution used on a $150 \mathrm{~cm}^{2}$ paper was $4 \mathrm{ml}$. Test boxes of $15 \times 10 \times 2 \mathrm{~cm}$ were placed in an incubator for 26 hours at $27^{\circ} \pm 1{ }^{\circ} \mathrm{C}$ with a 1,100 lux light. In the second test insecticide solutions were sprayed directly on bees. After treatment the bees were allowed to forage on sweet clover (Melilotus alba) in recovery cages. Levels utilized are shown in table 2.

In the third test sweet clover plants were sprayed with insecticides, dried and introduced into cages of $1,5 \times 1,20 \times 0,75 \mathrm{~m}$ with megachiles.

Repellency was tested on females. This experiment took place in a greenhouse with sweet clover. Half the number of plants were treated either with decamethrin or fenvalerate and the other half were control plants.

\section{RESULTS}

Knock-down effect was nearly as strong in fenvalerate as in decamethrin. In the case of decamethrin $44 \%$ of bees were affected by detoxication and with fenvalerate $59 \%$ (Fig. 1). When the exposure on treated paper lasted up to 4 hours (Fig. 2) knock-down effect and toxicity were similar for both pyrethroids. According to Fig. 1 and Fig. 2 the classification of the compounds from high to low poisoning level was as follows : bromophos, phosalone, decamethrin, fenvalerate, oxydemeton-methyl.

Speed of action of these insecticides was the same as in the first test when used in a direct spray (Fig. 3). The toxic materials were: bromophos and decamethrin. Phosalone, oxydemeton-methyl and fenvalerate were moderately toxic. Detoxication was considerably high in fenvalerate $(62 \%)$.

Three compounds proved to be highly toxic to bees after 5 days of foraging in cages (Fig. 4): bromophos, phosalone, decamethrin. Two caused moderate and low mortality : oxydemeton-methyl and fenvalerate, respectively.

In greenhouse repellency was noted for 1 hour and $15 \mathrm{mn}$ with fenvalerate and 3 hours and $30 \mathrm{mn}$ with decamethrin (Table 3). 


\section{Discussion-conclusion}

Males of megachiles were highly or moderately susceptible to two insecticides (bromophos and phosalone) reputed to be non toxic to honey bee, but they were slightly affected by oxydemetonmethyl. The pyrethroids are classified as slightly or highly toxic to bees as decamethrin and as slightly or moderately toxic as fenvalerate.

Adverse effects of oxydemeton-methyl were observed only when nectar was consumed.

Knock-down effect of pyrethroids could cause some trouble in the nesting behaviour of megachiles, even after detoxication. Further research in this field should be carried out.

We intend to compare male and female susceptibility in the same test conditions and to estimate pyrethroids persistence.

\section{BIBLIOGRAPHIE}

Bournoville R., 1979. - Les insectes nuisibles à la production de semences de luzerne. Inventaire, éléments biologiques orientant la lutte. Bull. F.N.A.M.S. Suppl. $\mathrm{n}^{\circ} 66,55-68$.

Cazenave A., Debray Ph., Esteulle M., 1980. - Étude du comportement d'abeilles Apis mellifera sur des plantes en fleurs traitées avec le fenvalérate. Com. 16-04-80 S.F.P.P.

Delaude A., 1981. - Une nouvelle race d'insecticides : les pyréthrinoïdes. Bull. F.N.A.M.S., $\mathrm{n}^{\circ}$ 73, 17-18.

Elliot M., JANes N. F., PotTer C., 1978. - The future of pyrethroids in insect control. Annu. Rev. Entorol. 23, 443-469.

GERIG V. L., 1979. - Bienengiftigkeit der synthetischen pyrethrine. Schweiz. Bienenztg. 5, 228-236.

JOHANSEN C. A., 1979. - Honey bee poisoning by chemicals : signs, contributing factors, current problems and prevention. Bee Wld. 60, 109-127.

Lhoste J., 1978. - Pyréthrines naturelles et pyréthrinoïdes de synthèse. Phytoma, juin, 9-13.

TASEI J.-N., 1977. - Méthode de test de toxicité des insecticides applicable aux abeilles solitaires et plus particulièrement à Megachile pacifica Pz. (Hym. megachilidae). Apidologie, 8, 129-139.

TASEI J.-N., 1977. - Action de quelques insecticides sur une abeille solitaire : Megachile pacifica $\mathrm{Pz}$. (Hym. megachilidae). Apidologie, 8, 111-127. 